\title{
Metabolic flux determination by stationary 13-C tracer experiments: Analysis of sensitivity, identifiability and redundancy
}

\author{
Wolfgang Wiechert \\ Institut für Biotechnologie, Forschungszentrum Jülich \\ 52425 Jülich, Germany. e-mail: w.wiechert@kfa-juelich.de
}

\begin{abstract}
Stationary ${ }^{13} \mathrm{C}$ tracer experiments supply a large amount of information related to metabolic fluxes in microorganisms. Unknown intracellular fluxes can be determined from some directly measured metabolic fluxes and the fractional labelling of intracellular carbon atom pools. To this end the algebraic flux and carbon balance equations have to be solved by parameter-fitting. The statistical quality of the results is judged by local (i.e. linearized) sensitivity analysis. On the other hand some generally applicable computer algebraic algorithms are given for global redundancy and identifiability analysis. They enable the dimension of the flux determination problem to be significantly reduced. As an application example some global results for the anaplerotic reaction section from the central metabolism are derived.
\end{abstract}

\section{Keywords}

Metabolic fluxes, isotope labelling, sensitivity, identifiability, redundancy, computer algebra

\section{INTRODUCTION}

The determination of intracellular metabolic fluxes in microorganisms is of great interest for biotechnology. A complete quantitation of all reaction rates in the central metabolic pathways allows the effect of genetic manipulations on the yield of certain desired metabolic products to be characterized in vivo. Moreover such a method will supply valuable information for a systematic approach to increase product formation by microorganisms (Bailey, 1991).

A recently developed method of determining intracellular fluxes is based on ${ }^{13} \mathrm{C}$ NMR labelling data in combination with direct measurements of the fluxes between the cell interior and the surrounding medium (Marx et al., 1995). The organism must therefore be in a metabolic steady state which can be maintained inside a bioreactor. In this situation a tracer substrate which is labelled at a certain carbon atom by ${ }^{13} \mathrm{C}$ is fed into the system. After an isotopically stationary steady state has been reached for all metabolic intermediates, the fractional amount of labelled carbon atoms within intracellular metabolites can be measured (Figure 1). For this purpose the 


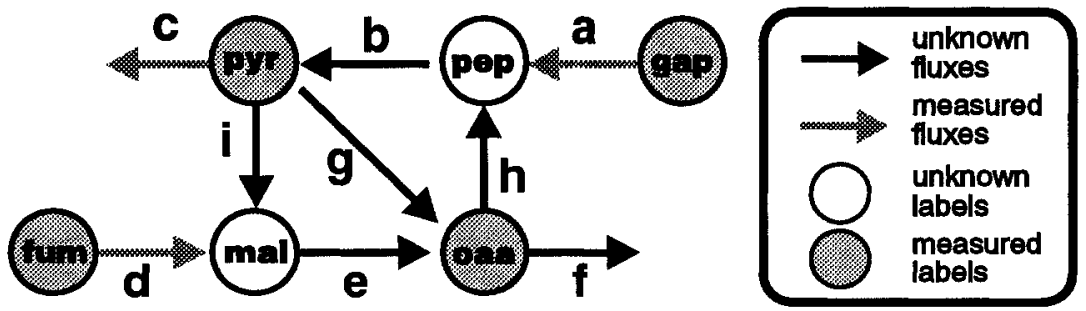

Figure 1 The anaplerotic reaction section in the central metabolism of $C$. glutamicum with the measurement data that can be supplied by a ${ }^{13} \mathrm{C}$ NMR tracer experiment. Upper row: glycolytic metabolites. Lower row: metabolites in the citric acid cycle.

labelled amino acids are extracted from the cell protein, which is the distinguishing feature of the new method.

This new technique supplies a previously unattainable amount of (stationary) measurement data from which the intracellular metabolite fluxes have to be determined. 15 fluxes and 23 fractional labels within $C$. glutamicum could be directly measured by Marx et al. (1995)! From this data set nearly all fluxes in cellular metabolism could be determined and additionally șeveral reverse fluxes (Wiechert et al., 1995).

However, since the corresponding model equations are nonlinear and the metabolic networks display a high degree of coupling, it cannot easily be judged whether the measurements contain sufficient information about the unknown fluxes (sensitivity and identifiability problem). On the other hand several time-consuming measurement procedures can be omitted if it turns out that certain data contain no additional information (redundancy problem). This contribution presents some generally applicable algorithms and an application for the treatment of identifiability and redundancy problems corresponding to metabolic carbon isotope labelling systems.

\section{EXAMPLE: ANAPLEROTIC REACTIONS}

The model equations will be introduced using a very simple example from the central metabolism of C. glutamicum. The anaplerotic section of the metabolism (as shown in Figure 1) was chosen because it serves to demonstrate a certain nonidentifiability problem that will be explained later on. More details concerning the formulation of model equations can be taken from (Wiechert $e t$ al., 1995).

Firstly, the state vectors are constructed as follows. All metabolic fluxes in the system are combined into the flux vector

$\mathbf{v}=(a, b, c, d, \epsilon, f, g, h, i)^{T}$

In our case all participating metabolites have 3 carbon atoms which do not change places in any of the reaction steps. For this reason we can restrict our study to the first carbon atom of each metabolite. The ${ }^{13} \mathrm{C}$ labelling fractions in each carbon atom pool are then given by the vectors

$\mathbf{x}^{\text {inp }}=(\text { gap }, \text { fum })^{T}$ and $\mathbf{x}=(p e p, p y r, o a a, m a l)^{T}$. 
Here $\mathbf{x}^{\text {inp }}$ corresponds to input metabolites with known labelling state while $\mathbf{x}$ corresponds to metabolic intermediates.

Secondly, some material balance equations hold because the system is in a stationary state. The metabolic fluxes connected with an intermediate pool add up to zero (linear stoichiometric equations), which can be used for term elimination:

$$
\begin{aligned}
& \text { pep: } a+h=b \quad b=a \quad+h \\
& \text { pyr: } b=c+g+i \Longrightarrow e=a-c+d-g+h \\
& \text { oaa: } e+g=f+h \quad \Longrightarrow \quad f=a-c+d \\
& \text { mal: } d+i=e \quad i=a-c-g+h
\end{aligned}
$$

Similarly, the isotope labelling balance equations reflect the fluxes of labelled carbon atoms. A metabolic flux $\mathbf{v}_{i}: \mathbf{x}_{j} \rightarrow \mathbf{x}_{k}$ carries the absolute amount of $\mathbf{v}_{i} \cdot \mathbf{x}_{j}$ of labelled material per time unit. This gives rise to a system of equations which is bilinear with respect to fluxes and labels:

$$
\left(\begin{array}{l}
0 \\
0 \\
0 \\
0
\end{array}\right)=\left(\begin{array}{cccc}
-b & \cdot & h & \cdot \\
b & -c-g-i & \cdot & \cdot \\
\cdot & g & -f-h & e \\
\cdot & i & \cdot & -e
\end{array}\right)\left(\begin{array}{l}
p e p \\
p y r \\
o a a \\
\text { mal }
\end{array}\right)+\left(\begin{array}{ll}
a & \cdot \\
\cdot & \cdot \\
\cdot & \cdot \\
\cdot & d
\end{array}\right)\left(\begin{array}{l}
\text { gap } \\
\text { fum }
\end{array}\right) .
$$

Using (1) the $4 \times 4$ system matrix in (2) further reduces to:

$$
\begin{aligned}
& \left(\begin{array}{cccc}
-a-h & \cdot & h & \cdot \\
a+h & -a-h & \cdot & \cdot \\
\cdot & g & -a+c-d-h & a-c+d-g+h \\
\cdot & a-c-g+h & \cdot & -a+c-d+g-h
\end{array}\right) \\
& =a \cdot\left(\begin{array}{rrrr}
-1 & . & \cdot & \cdot \\
1 & -1 & . & . \\
\cdot & \cdot & -1 & \mathbf{1} \\
\cdot & 1 & \cdot & -1
\end{array}\right)+c \cdot\left(\begin{array}{rrrr}
\cdot & \cdot & \cdot & \cdot \\
\cdot & \cdot & \cdot & . \\
\cdot & \cdot & 1 & -1 \\
\cdot & -1 & \cdot & 1
\end{array}\right)+d \cdot\left(\begin{array}{cccc}
\cdot & \cdot & \cdot & \cdot \\
\cdot & \cdot & \cdot & . \\
\cdot & \cdot & -1 & 1 \\
\cdot & \cdot & \cdot & -1
\end{array}\right)+\ldots
\end{aligned}
$$

From now on $\mathrm{v}$ is replaced by the reduced flux vector $\mathrm{v}=(a, c, d, h, g)^{T}$.

\section{GENERAL STRUCTURE OF MODEL EQUATIONS}

Collecting all integer coefficients associated with a carbon flux $\mathbf{v}_{i}$ into the atom transition matrices $\mathbf{P}_{i}, \mathbf{P}_{i}^{\text {inp }}$ as has been done in (3) the general algebraic structure of the metabolic carbon isotope labelling system (2) is comprehensively given by:

$\mathbf{0}=\left(\sum_{i=1}^{\operatorname{dim} \mathbf{v}} \mathbf{v}_{i} \cdot \mathbf{P}_{i}\right) \cdot \mathbf{x}+\left(\sum_{i=1}^{\operatorname{dim} \mathbf{v}} \mathbf{v}_{i} \cdot \mathbf{P}_{i}^{\text {inp }}\right) \cdot \mathbf{x}^{\mathrm{inp}} \stackrel{\text { def }}{=} \mathbf{P}(\mathbf{v}) \cdot \mathbf{x}+\mathbf{P}^{\mathrm{inp}}(\mathbf{v}) \cdot \mathbf{x}^{\mathrm{inp}}$

This is sufficient to understand the mathematical problems under consideration. For a reasonably complex metabolic network $\operatorname{dim} v \approx 30$ and $\operatorname{dim} x \approx 100$ can be reached, which makes clear that identifiability and redundancy are indeed nontrivial problems. For 'nonpathological' systems 
the matrix $\mathbf{P}(\mathbf{v})$ can be proven to be positively definite (Anderson, 1983) so that we obtain a well defined solution

$\mathbf{x}=\mathbf{x}(\mathbf{v})=-\mathbf{P}(\mathbf{v})^{-1} \cdot \mathbf{P}^{\text {inp }}(\mathbf{v}) \cdot \mathbf{x}^{\mathrm{ipp}}$.

Several of the fluxes $\mathbf{v}_{i}$ as well as the carbon atom labels $\mathbf{x}_{j}$ can be directly measured (see Figure 1). This is formally described by coordinate projection matrices $\mathbf{M}$ (i.e. matrices composed of unit vectors). The measurement noise $\varepsilon$ is assumed to be normally distributed with expectation $\mathbf{0}$ and known covariance matrix $\boldsymbol{\Sigma}$ giving rise to the measurement equations:

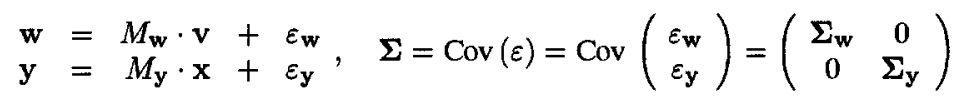

\section{FLUX ESTIMATION AND SENSITIVITY ANALYSIS}

Usually, there are more measured quantities than unknown fluxes. The problem of flux determination thus is an inverse problem associated with equation (5) and (6). It can be treated numerically by computing a least squares estimate with respect to the appropriate weighted vector norms $\|\mathbf{x}\| \mathbf{\Sigma}=\mathrm{x}^{T} \cdot \mathbf{\Sigma}^{-1} \cdot \mathbf{x}$ :

$\hat{\mathbf{v}}=\arg \min _{\mathbf{v}}\left\|\mathbf{w}-M_{\mathbf{w}} \cdot \mathbf{v}\right\|_{\mathbf{\Sigma}_{\mathbf{w}}}^{2}+\left\|\mathbf{y}-M_{\mathbf{y}} \cdot \mathbf{x}(\mathbf{v})\right\|_{\mathbf{\Sigma}}^{2}$

The statistical quality of the obtained flux estimate $\hat{v}$ can be judged by computing sensitivity measures and confidence bounds. The key to the solution of these problems is obtained by implicit differentiation of equation (4):

$$
\frac{\partial \mathbf{x}}{\partial \mathbf{v}_{j}}(\mathbf{v})=-\mathbf{P}(\mathbf{v})^{-1} \cdot\left(\mathbf{P}_{j} \cdot \mathbf{x}+\mathbf{P}_{j}^{\mathrm{inp}} \cdot \mathbf{x}^{\mathrm{inp}}\right)
$$

This shows that the same matrix inversion serves for both the computation of $x$ and its derivatives with respect to $\mathrm{v}$, which is also of great use for an efficient iterative solution of the least squares problem (7). Using (8) and a standard Gauß-Newton linearization argument (Seber, Wild, 1989), it is merely a technical problem to compute the combined partial derivative $\partial(\mathbf{w}, \mathbf{y}) / \partial \mathbf{v}$ (on the assumption that no noise is present, i.e. $\varepsilon=0$ ) and from this:

$$
\begin{aligned}
& \text { the weighted output sensitivity } \operatorname{Sens}_{\mathbf{v}}^{\mathbf{w}, \mathbf{y}}(\mathbf{v})=\sqrt{\Sigma}^{-1} \cdot \frac{\partial(\mathbf{w}, \mathbf{y})}{\partial \mathbf{v}}(\mathbf{v}) \\
& \text { the estimator's covariance matrix } \operatorname{Cov}(\hat{\mathbf{v}}) \approx\left[\operatorname{Sens}_{\mathbf{v}}^{\mathrm{w}, \mathrm{y}}(\hat{\mathbf{v}})^{T} \cdot \operatorname{Sens}_{\mathrm{v}}^{\mathrm{w}, \mathrm{y}}(\hat{\mathbf{v}})\right]^{-1} \\
& \text { and the weighted estimator's sensitivity } \operatorname{Sens}_{\mathbf{w}, \mathbf{y}}^{\hat{v}} \approx \operatorname{Cov}(\hat{\mathbf{v}}) \cdot \operatorname{Sens}_{\mathbf{v}}^{\mathbf{w}, \mathbf{y}}(\hat{\mathbf{v}})^{T}
\end{aligned}
$$

The convergence of the parameter-fitting algorithm that has been applied to the data set in (Marx et al., 1995) can be taken as an indicator of flux identifiability from the measured data. Sensitivity analysis then helped to detect redundant data sources and identifiability problems (these results will be presented elsewhere). However, they rely on a linearization argument and thus are purely local in nature. Moreover they represent a posteriori results in contrast to a priori results that make no use of concrete measurement data. To this end some generally applicable 
computer algebraic methods for global system analysis will now be presented. Finally, they will be applied to solve the already mentioned nonidentifiability problem that occured in the anaplerotic section.

\section{IDENTIFIABILITY AND REDUNDANCY ANALYSIS}

Firstly, a precise definition of the terms 'identifiability' and 'redundancy' has to be given. Aiming at the application of computer algebraic methods we will assume from now on that $w, y$ can be measured without any error (i.e. $\varepsilon_{\mathbf{w}}=\varepsilon_{\mathbf{y}}=0$ ). Furthermore it will be assumed for simplicity that the entries of $\mathbf{v}, \mathbf{x}$ have been ordered such that the last components are the measured ones. Introducing the vectors $\mathbf{u}, \mathbf{z}$ of unknown fluxes or labels we get:

$\mathbf{v}=\left(\begin{array}{l}\mathbf{u} \\ \mathbf{w}\end{array}\right)=\left(\begin{array}{r}\mathbf{u} \\ \mathbf{M}_{\mathbf{w}} \cdot \mathbf{v}\end{array}\right) \quad$ and $\quad \mathbf{x}=\left(\begin{array}{l}\mathbf{z} \\ \mathbf{y}\end{array}\right)=\left(\begin{array}{r}\mathbf{z} \\ \mathbf{M}_{\mathbf{y}} \cdot \mathbf{x}\end{array}\right)$

The set of all flux and label vectors producing the observed output $(\mathbf{w}, \mathbf{y})$ then is

$\boldsymbol{\Omega}(\mathbf{w}, \mathbf{y}) \stackrel{\text { def }}{=}\left\{(\mathbf{v}, \mathbf{x}) \mid \mathbf{P}(\mathbf{v}) \cdot \mathbf{x}+\mathbf{P}^{\text {inp }}(\mathbf{v}) \cdot \mathbf{x}^{\text {inp }}=0 \wedge \mathbf{M}_{\mathbf{w}} \mathbf{v}=\mathbf{w} \wedge \mathbf{M}_{\mathbf{y}} \mathbf{x}=\mathbf{y}\right\}$

Identifiability of $\mathbf{v}$ now precisely means that $\boldsymbol{\Omega}(\mathbf{w}, \mathbf{y})$ contains exactly one element.

Clearly, if the unknown fluxes $u$ can be uniquely determined from $(\mathbf{w}, \mathbf{y})$, any further measurement data are redundant with $(\mathbf{w}, \mathbf{y})$ by $(5)$. However, it is desirable to decide redundancy independently from the identifiability problem. By a redundancy relation we denote some parametrized equation of type $f_{(\mathbf{w}, \mathbf{y})}(\mathbf{v}, \mathbf{x})=\mathbf{0}$ that holds for all $(\mathbf{v}, \mathbf{x}) \in \boldsymbol{\Omega}(\mathbf{w}, \mathbf{y})$.

As proven by Wiechert and Schwingenheuer (1995) the identifiability and redundancy problems can be almost completely solved using the Gröbner base algorithm from polynomial ideal theory. However, the computational complexity of this algorithm is much too great to be applied to complex metabolic networks. For this reason, efficient methods are required for further complexity reduction. To this end we will restrict ourselves to linear algebraic methods that enable linear redundancy relations of type

$f_{(\mathbf{w}, \mathbf{y})}(\mathbf{v}, \mathbf{x})=\eta(\mathbf{w}, \mathbf{y})+\sum_{i=1}^{\operatorname{dim} \mathbf{v}} \eta_{i}^{\mathbf{v}}(\mathbf{w}, \mathbf{y}) \cdot \mathbf{v}_{i}+\sum_{i=1}^{\operatorname{dim} \mathbf{x}} \eta_{i}^{\mathbf{x}}(\mathbf{w}, \mathbf{y}) \cdot \mathbf{x}_{i}=0 \quad \forall(\mathbf{v}, \mathbf{x}) \in \mathbf{\Omega}(\mathbf{w}, \mathbf{y})$

to be constructed. Clearly, each relation of this type can be considered for dimension reduction of $\mathbf{u}$ or $\mathbf{z}$, which simultaneously helps to treat the identifiability problem. Consequently, a combination of dimension reduction steps with the Gröbner base algorithm has the potential to treat even complex networks.

\section{COMPUTER ALGEBRAIC ALGORITHMS}

As a preliminary equation (5) can be reformulated using Cramer's rule:

$\mathbf{x}_{i} \cdot \operatorname{det} \mathbf{P}(\mathbf{v})=-\operatorname{det}\left(\mathbf{P}(\mathbf{v}) \square \mathbf{P}^{\text {inp }}(\mathbf{v}) \cdot \mathbf{x}^{\text {inp }}\right), \quad i=1, \ldots, \operatorname{dim} \mathbf{x}$ 
where $i$ denotes replacement of the $i$ th column by the vector on the right side. All entries of $\mathbf{P}(\mathbf{v})$ and $\mathbf{P}^{\text {inp }}(\mathbf{v}) \cdot \mathbf{x}^{\text {inp }}$ are linear combinations of fluxes $\mathbf{v}_{i}$. Consequently, both sides of (10) are polynomials of at most degree $\operatorname{dim} \mathbf{x}$ with respect to the $\mathbf{v}_{i}$. Now separating the unknown part $\mathbf{u}$ from the known part $\mathbf{w}$ of $\mathbf{v}$ and using multi-index notation (i.e. $\mathbf{u}^{\alpha}=\mathbf{u}_{1}^{i_{1}} \cdot \ldots \cdot \mathbf{u}_{m}^{i_{m}}, \alpha=$ $\left.\left(i_{1}, \ldots, i_{m}\right), \# \alpha=i_{1}+\ldots+i_{m}\right)$ the left and right sides in equation (10) can be rewritten in the form

$\mathbf{x}_{i} \cdot \sum_{\# \alpha \leq \operatorname{dim} \mathbf{x}} c^{\alpha}(\mathbf{w}) \cdot \mathbf{u}^{\alpha}=-\sum_{\# \alpha \leq \operatorname{dim} \mathbf{x}} c_{i}^{\alpha}\left(\mathbf{w}, \mathbf{x}^{\mathrm{inp}}\right) \cdot \mathbf{u}^{\alpha}, \quad i=1, \ldots, \operatorname{dim} \mathbf{x}$

with certain polynomials $c^{\alpha}, c_{i}^{\alpha}$ that do not depend on $\mathbf{u}$. Assume now that a linear relation of type

$\eta=\sum_{i=1}^{\operatorname{dim} \mathbf{x}} \eta_{i}^{\mathbf{x}} \cdot \mathbf{x}_{i} \Longleftrightarrow \eta \cdot \operatorname{det} \mathbf{P}(\mathbf{v})=\sum_{i=1}^{\operatorname{dim} \mathbf{x}} \eta_{i}^{\mathbf{x}} \cdot \mathbf{x}_{i} \cdot \operatorname{det} \mathbf{P}(\mathbf{v})$

holds for all $u$. Then we have from (10) and (11):

$\eta \cdot \sum_{\# \alpha \leq \operatorname{dim} \mathbf{x}} c^{\alpha}(\mathbf{w}) \cdot \mathbf{u}^{\alpha}=-\sum_{i=1}^{\operatorname{dim} \mathbf{x}} \eta_{i}^{\mathbf{x}} \cdot \sum_{\# \alpha \leq \operatorname{dim} \mathbf{x}} c_{i}^{\alpha}\left(\mathbf{w}, \mathbf{x}^{\operatorname{inp}}\right) \cdot \mathbf{u}^{\alpha}$

This is a polynomial equation with respect to $u$ and henceforth all coefficients corresponding to the same multi-index $\alpha$ must be equal. This leads to

Algorithm 1 Compute a priori linear redundancy relations with respect to $\mathbf{x}$.

Step 1: Compute all polynomials $c^{\alpha}(\mathbf{w}), c_{i}^{\alpha}\left(\mathbf{w}, \mathbf{x}^{\mathrm{inp}}\right)$ for $\# \alpha \leq \operatorname{dim} \mathbf{u}$.

Step 2: For each $\alpha$ a linear equation $\eta \cdot c^{\alpha}(\mathbf{w})=-\sum_{i=1}^{\operatorname{dim} \mathbf{x}} \eta_{i}^{\mathbf{x}} \cdot c_{i}^{\alpha}\left(\mathbf{w}, \mathbf{x}^{\mathrm{inp}}\right)$ holds. Use this equation set to determine a basis for the space of all equations of type (12).

Clearly, steps 1 and 2 should be performed in succession for each new $\alpha$. Because there are usually many more equations than variables $\eta, \eta_{i}^{\mathbf{X}}$, most generated equations will be trivial (i.e. $c^{\alpha}=0 \wedge \forall_{i} c_{i}^{\alpha}=0$ ) or redundant with the already computed solutions. A simple stopping criterion for this process (that cannot be given here for shortness) can be computed numerically using a Monte Carlo method. Moreover, efficient algorithms for symbolic determinant evaluation are discussed by Gielen and Sansen (1991).

The next algorithm determines mixed linear relations for $u, z$. To this end we split the atom transition matrices $\mathbf{P}_{i}$ from equation (4) into two parts $\mathbf{Q}_{i}, \mathbf{R}_{i}$ such that

$\mathbf{P}_{i} \cdot \mathbf{x}=\left(\mathbf{Q}_{i} \mathbf{R}_{i}\right) \cdot\left(\begin{array}{c}\mathbf{z} \\ \mathbf{y}\end{array}\right)=\mathbf{Q}_{i} \cdot \mathbf{z}+\mathbf{R}_{i} \cdot \mathbf{y}, i=1, \ldots, \operatorname{dim} \mathbf{v}$.

Rearranging all completely unobservable bilinear terms $\mathbf{u}_{i} \cdot \mathbf{z}_{j}$ in (4) to the left side we get

$-\sum_{i=1}^{\operatorname{dim} \mathbf{u}} \mathbf{u}_{i} \cdot \mathbf{Q}_{i} \cdot \mathbf{z}=\sum_{i=1}^{\operatorname{dim} \mathbf{w}} \mathbf{w}_{i} \cdot \mathbf{Q}_{i+\operatorname{dim} \mathbf{u}} \cdot \mathbf{z}+\sum_{i=1}^{\operatorname{dim} \mathbf{v}} \mathbf{v}_{i} \cdot \mathbf{R}_{i} \cdot \mathbf{y}+\sum_{i=1}^{\operatorname{dim} \mathbf{u}} \mathbf{v}_{i} \cdot \mathbf{P}_{i}^{\mathrm{inp}} \cdot \mathbf{x}^{\mathrm{inp}}$ 
This leads us to

Algorithm 2 Compute a priori linear redundancy relations with respect to $\mathbf{u}, \mathbf{z}$.

Step 1: Determine all matrices $\mathbf{Q}_{i}, i=1, \ldots, \operatorname{dim} \mathbf{u}$.

Step 2: Determine an annihilation matrix $\mathbf{A}$ fulfilling $\mathbf{A}^{T} \cdot \mathbf{Q}_{i}=\mathbf{0}$ for $i=1, \ldots, \operatorname{dim} \mathbf{u}$ by computing a vector space complement of all column vectors of $\mathbf{Q}_{1}, \ldots, \mathbf{Q}_{\mathrm{dimu}}$.

Step 3: Multiply equation (4) with A from the left thus producing a set of linear equations with respect to $\mathbf{u}, \mathbf{z}$.

Since the matrices $\mathbf{P}_{i}, \mathbf{P}_{i}^{\text {inp }}$ are usually sparsely populated there is a good chance that this equation set is nontrivial. Clearly, this chance increases with the number of measured fluxes and labels.

\section{APPLICATION EXAMPLE}

Both algorithms will now be applied to the anaplerotic example. In (Marx et al., 1995) the peripheral fluxes $a, c, d$ could be indirectly estimated from the overall data set. Additionally, the fractional intermediary labels pyr, oaa and the input labels gap, fum could be measured (see Figure 1). By equation (1) this leaves only 2 degrees of freedom (represented by $g, h$ ) in the flux system.

Algorithm 1 is now used to compute all linear redundancy equations of type (12), i.e. $\eta=$ $\eta_{p e p} \cdot p e p+\eta_{p y r} \cdot p y r+\eta_{o a a} \cdot o a a+\eta_{m a l} \cdot m a l$ with coefficients depending only on measured parameters. This vector space turns out to be 2 -dimensional as determined by the equations

$0=\eta_{m a l}, \quad \eta=-\frac{a \cdot g a p+d \cdot f u m}{a-c+d} \cdot \eta_{o a a}, \quad 0=\eta_{p e p}+\eta_{p y r}+\frac{c}{a-c+d} \cdot \eta_{o a a}$.

As can easily be verified these conditions are fulfilled by the linearly independent equations

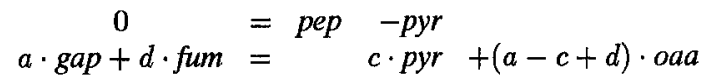

The first equation holds independently of the parameters $a, c, d$. This shows that it is never useful to measure both pyr and pep. The second equation turns out to be the systems overall label balance (remember that $a-c+d=f$ by (1)). As a result it is superfluous to measure oaa too. For dimensional reasons it follows that $f, g$ cannot be simultaneously determined without further measurements.

Applying Algorithm 2 requires to compute the left side matrix of equation (13)), which is

$$
-\sum_{i=1}^{\operatorname{dim} \mathbf{u}} \mathbf{u}_{i} \cdot \mathbf{Q}_{i}=h \cdot\left(\begin{array}{rr}
-1 & \cdot \\
1 & \cdot \\
\cdot & 1 \\
\cdot & -1
\end{array}\right)+g \cdot\left(\begin{array}{rr}
\cdot & \cdot \\
\cdot & \cdot \\
\cdot & -1 \\
\cdot & 1
\end{array}\right) \Longrightarrow \mathbf{A}^{T}=\left(\begin{array}{llll}
1 & 1 & 0 & 0 \\
0 & 0 & 1 & 1
\end{array}\right) .
$$


Multiplication of (2) with $\mathbf{A}^{T}$ yields (use (3)):

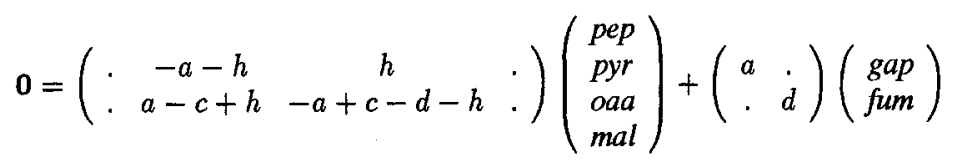

From this two alternative solutions for $h$ are obtained (which of course must be equal):

$h_{1}=a \cdot \frac{p y r-g a p}{o a a-p y r}, \quad h_{2}=(c-a)-d \cdot \frac{o a a-f u m}{o a a-p y r}$

Consequently, $g$ is not identifiable from the measured data, which explains the identifiability problem that occurred with the data in (Marx et al., 1995). It should be pointed out that the equality $h_{1}=h_{2}$ produces exactly the already mentioned overall label balance in (14). On the other hand pep $=p y r$ is not reproduced with Algorithm 2. This shows that both algorithms have a different scope.

Although $\mathrm{mal}$ is currently not measurable it should be mentioned that $g$ is identifiable in this situation. Algorithm 2 then produces: $m a l=(h+a-c+d) \cdot(o a a-m a l) /(p y r-m a l)$

\section{REFERENCES}

Anderson, D.H. (1983) Compartmental Modelling and Tracer Kinetics, volume 50 of Lecture notes in Biomathematics. Springer.

Bailey, J.E. (1991) Towards a science of metabolic engineering. Science, 252, 1668-75.

Gielen, G. and Sansen, W. (1991) Symbolic Analysis for Automated Design of Analog Integrated Circuits. Kluwer Academic Publishers.

Marx A., de Graaf A.A., Wiechert W., Eggeling L. and H. Sahm (1995). Determination of the Fluxes in Central Metabolism by ${ }^{13}$ C NMR Combined with Metabolite Balancing. Submitted.

Seber, G.A.F. and Wild, C.J. (1989) Nonlinear Regression. Wiley.

Wiechert W., de Graaf A.A. and Marx A. (1995) In Vivo Stationary Flux Determination Using ${ }^{13} \mathrm{C}$ NMR Isotope Labelling Experiments, in CAB 6, Computer Applications in Biotechnology (ed. K. Schügerl and A. Munack), Pergamon. In Press.

W. Wiechert and V. Schwingenheuer. Algebraic methods for the analysis of redundancy and identifiability in metabolic ${ }^{13} \mathrm{C}$ labelling systems. 1995. in Bioinformatik 1995 (ed. D. Schomburg). In Press. 